\title{
DIETAS DE TERMINAÇÃO DE FRANGOS DE CORTE SUPRIMIDOS DA SUPLEMESTTAÇÃO MINERAL E VITAMÍNICA
}

\author{
Finishing diets of the broilers without mineral and vitamin supplementation \\ Marcos Rosniecek, Aline Félix Schneider, Thiago El Hadi Perez Fabregat, Vladimir \\ de Oliveira, Clóvis Eliseu Gewehr ${ }^{1}$ \\ ${ }^{1}$ Correspondência: Clóvis Eliseu Gewehr: a2ceg@cav.udesc.br
}

\begin{abstract}
RESUMO: O objetivo deste trabalho foi avaliar o desempenho de frangos de corte de submetidos a dietas de terminação com a retirada do suplemento mineral e vitamínico (premix). Foram utilizados 480 frangos de corte, machos, de linhagem comercial, de 42 a 49 dias, distribuídos em quatro tratamentos determinados pelo dia de retirada do premix mineral e vitamínico da dieta, aos 42, 44, e 46 dias, comparados a um controle em que não ocorreu a retirada do premix. Utilizou-se um delineamento inteiramente casualizado com seis repetições de 20 aves. Os resultados indicaram que consumo de ração (g) e mortalidade (\%) não foram alterados $(P>0,10)$ pelos dias que anteciparam a retirada do premix das dietas, entretanto o ganho de peso $(\mathrm{g})$, conversão alimentar $(\mathrm{kg} / \mathrm{kg})$ e viabilidade econômica foram afetados $(P<0,001)$ linearmente. A retirada do suplemento mineral $e$ vitamínico da ração de terminação de frangos de cortes reduz o desempenho zootécnico sem alterar o consumo alimentar, entretanto aumenta o custo de produção na fase de terminação.
\end{abstract}

Palavras-chave: Avicultura; nutrição animal; premix; ração

\section{Finishing diets of the broilers without mineral and vitamin supplementation}

ABSTRACT: The objective of this study was evaluate the broiler performance of fed diets termination with the removal of vitamin and mineral supplement (premix). Were utilized 480 commercial broiler male at 42 to 49 days, distributed in four treatments, which were determined by removal of the vitamin and mineral supplement 42, 44, and 46 days compared to a control it did not occur to withdrawal of premix. Was used a completely randomized design with six replicates of 20 birds each. The results of the analysis of variance indicated that feed intake $(\mathrm{g})$ and mortality (\%) were not affected $(P>0.10)$ for the days that anticipated the withdrawal of premix diets , however weight gain $(\mathrm{g})$, feed conversion $(\mathrm{g} / \mathrm{g})$ and economic viability were highly affected linearly $(P<0.001)$. The removal of vitamin and mineral supplement of the finishing diet of the broilers reduces growth performance with no effects in feed intake, however increases the production cost in the finishing phase.

Key Words: poultry ; animal nutrition; premix ; feed 


\section{INTRODUÇÃO}

A seleção genética que os frangos de corte modernos vêm sendo submetidos em busca de melhorias no ganho de peso e rendimento de carcaça faz com que os nutricionistas tenham que rever constantemente os efeitos deste processo sobre a nutrição. A alimentação é o item que mais onera a criação de frangos e, neste contexto, estratégias nutricionais para racionalizar o uso dos alimentos nas dietas podem acarretar em redução dos custos de produção trazendo benefícios para esta cadeia produtiva. É constante o desafio da indústria avícola em produzir frangos de forma mais eficiente para reduzir o custo de produção, tornando-o ainda mais atraente para os consumidores.

Entre os componentes utilizados na formulação de rações estão os suplementos e aditivos, usualmente denominados de premix, cujo preço pode atingir cerca de $10 \%$ do custo total da alimentação de frangos de corte, conforme a quantidade e os tipos de ingredientes utilizados na sua produção (Ariki e Silva, 1977). O premix na sua essência é composto por vitaminas e microminerais e são considerados nutrientes fundamentais nas dietas de animais de rápido desempenho. Devem estar inclusas em quantidades corretas para permitir que as aves possam exprimir 0 seu potencial genético. Entretanto, alguns estudos têm demonstrando que estes nutrientes, em determinadas condições, podem estar sendo suplementados em excesso, levando em consideração uma margem de segurança (Rutz et al, 2002). Os níveis de vitaminas lipossolúveis podem superar até 25 vezes as recomendações e os das hidrossolúveis até cinco vezes, havendo grande variação dos níveis de vitaminas empregados comercialmente para frangos (Leeson, 2007).
A retirada do premix na fase de terminação dos frangos de corte pode ser uma alternativa para reduzir o custo de produção destes animais. Esta prática se fundamenta no fato que durante a fase de crescimento as aves recebem uma quantidade considerável destes nutrientes, sendo que os sintomas de deficiência na fase de terminação podem ser pouco evidentes. Os alimentos utilizados nas formulações dos frangos possuem uma determinada quantidade de vitaminas e micro-minerais, além disto, o fígado é um órgão que possui relativa capacidade de reserva destes nutrientes (Boleli et al., 2002) e não se sabe ao certo, quanto tempo estas reservas podem suprir a deficiência das dietas. A tentativa de redução da suplementação das vitaminas e microminerais nas dietas de terminação de frangos de corte tem sido objeto de vários estudos (Deyhim et al., 1990; Skinner et al., 1992; Christmas et al., 1995; Patel et al., 1997; Maiorka et al., 2002; Shahrasb et al. 2011; Ebrahimnezhad et al., 2011; Shahrasb, et al., 2012; Rahman et al., 2012 e Oba et al., 2012), entretanto ao observar os experimentos verifica-se que não há consenso nos resultados obtidos por estes pesquisadores, o que desperta 0 interesse para elucidar esta questão.

A hipótese deste trabalho é de que é possível a retirada do premix mineral e vitamínico alguns dias que antecedem o abate, pois as aves reduzem consideravelmente a necessidade destes nutrientes na fase de terminação e a quantidade contida nos alimentos juntamente com uma reserva hepática ou corporal podem atender a demanda por alguns dias. Neste contexto, este trabalho tem o objetivo de avaliar 0 desempenho zootécnico e o custo econômico de frangos de corte com a retirada do suplemento mineral $e$ vitamínico (premix) em dietas de terminação de frangos de corte. 


\section{MATERIAL E MÉTODOS}

Foram utilizados 480 frangos, machos, da linhagem Cobb, criados entre 42 e 49 dias de idade em aviário experimental aberto com cortinas plásticas nas laterais, com parcelas experimentais de $2 \mathrm{~m}^{2}$ separadas por tela, cada uma equipada com um comedouro tubular e um bebedouro pendular e cama de maravalha $(10 \mathrm{~cm})$ para recobrir o piso de cimento. Aos 42 dias, os frangos foram pesados individualmente e selecionados $(3,24 \mathrm{~kg} \pm 3 \%)$, distribuindoos aleatoriamente nas parcelas experimentais. Duas dietas de terminação (Tabela 1) foram formuladas a base de milho e farelo de soja de acordo com as recomendações de Rostagno et al. (2011). Ambas as dietas foram isonutritivas, sendo que uma dieta teve adição de premix e outra não teve a inclusão deste suplemento.

Desta forma os tratamentos foram ajustados para que as aves tivessem a retirada do premix de suas dietas aos 42 , 44 e 46 dias de idade, sendo que na dieta controle não foi retirado o suplemento durante o período experimental (42 a 49), ou seja, as aves receberam dietas sem premix durante os últimos sete, cindo, três e zero dias. A água e as dietas foram fornecidas a vontade em todos os tratamentos.

\begin{tabular}{lccccccc}
\multicolumn{3}{c}{ Tabela 1- Composição das dietas experimentais na fase de terminação de frangos } \\
de corte.
\end{tabular}

Foram avaliados consumo de ração (g/ave/dia), ganho de peso diário (g/ave), conversão alimentar $(\mathrm{g} / \mathrm{g})$, mortalidade (\%) e a viabilidade econômica. O consumo foi avaliado através da soma das quantidades consumidas das dietas com e sem a inclusão de premix de cada tratamento durante os sete dias de avaliação, dividindo-se pelo número total de aves das parcelas. A viabilidade econômica foi avaliada através do índice bio-econômico (IBE), desenvolvido por Guidoni et al. (1994) calculado pela fórmula:

IBE =ganho de peso $(g)-(R \$ k g$ de ração: $\mathrm{R} \$ \mathrm{~kg}$ ave viva) $x$ consumo de ração/ave (g).

O valor do quilo de ração está especificado na Tabela 1 e o preço do kg da ave viva foi considerado $R \$ 2,63$, cotados no mês de outubro de 2013 em uma agroindústria de Santa Catarina.

Utilizou-se um delineamento inteiramente casualizado, com quatro tratamentos em seis repetições (unidades experimentais) de 20 aves. Os resultados foram submetidos a análise de variância e quando observado diferença, as médias foram comparadas pelo teste SNK (5\%). Também foi 
procedida a análise de regressão para observar o comportamento dos tratamentos de acordo com a idade aves em cada item avaliado.

\section{RESULTADOS}

Consumo de ração (CR) e mortalidade não foram afetados $(P>0,10)$ pela retirada do suplemento mineral $\mathrm{e}$ vitamínico no período de 42 a 49 dias. Entretanto, o ganho de peso diário (GPD), conversão alimentar (CA) e índice viabilidade econômica (IBE) foram influenciados $(P<0,001)$ pelas dietas. Os resultados dos efeitos principais são apresentados na Tabela 2.

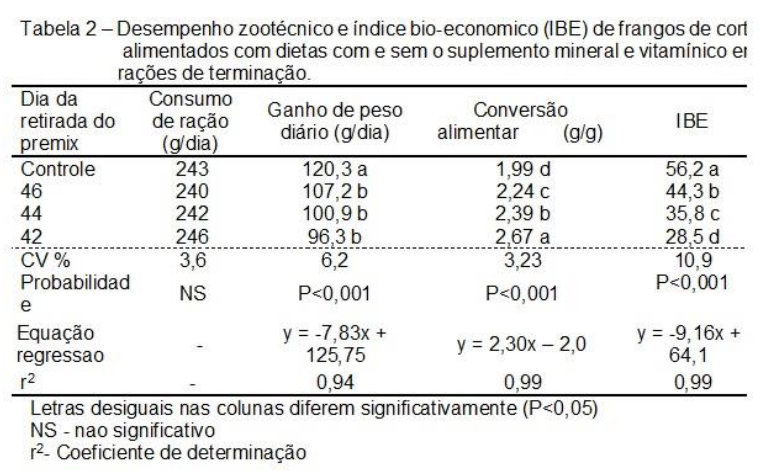

No GPD, nas dietas em que foram retirados o suplemento de vitaminas e micro-minerais ocorreu diferença $(P<0,001)$ em relação a dieta controle, entretanto nas dietas que tiveram a retirada do suplemento, o GPD foi semelhante $(P>0,05)$. $\mathrm{Na} C A$ e IBE, todos os tratamentos diferenciaram-se entre si $(P<0,001)$.

A análise de regressão (Tabela 2) indicou um comportamento linear $(\mathrm{P}<0,001)$ decrescente para GPD ( $\mathrm{r} 2=$ $0,941)$ e IBE $(r 2=0,987)$ e crescente para CA ( $r 2=0,989)$ em função da substituição da dieta com premix pela dieta sem premix de acordo com a idade das aves.

\section{DISCUSSÃO}

A mortalidade obtida entre as dietas (média geral de 0,41\%) ficou dentro da expectativa para a idade, sendo que Moravej et al. (2012a,b) obtiveram resultados similares na fase final com a retirada do suplemento de 36 a 42 dias. Khajali et al. (2006) e Shahrasb e Gerami (2011) indicam que a resposta imunológica das aves não é afetada pela retirada do premix na fase de terminaçao, onde uma imunidade reduzida poderia refletir na mortalidade. Não foram encontrados trabalhos indicando efeito negativo da retirada do suplemento micromineral e vitamínico da ração de terminação sobre a mortalidade, exceto quando 0 suplemento foi retirado de 28 a 49 dias (Deyhim e Teeter, 1993).

O CR das dietas ficou próximo daquele indicado pelo manual da linhagem (Cobb, 2012) que é $246 \mathrm{~g}$ para o período de 42 a 49 dias. O fato de não haver diferença no CR entre as dietas, provavelmente está relacionado com a limitação da capacidade física do trato digestório. O suplemento mineral e vitamínico é inserido nas rações em uma quantidade reduzida $(0,2 \%)$ e uma ave ingerindo $243 \mathrm{~g} / \mathrm{dia}$ (média dos tratamentos no período avaliado), corresponde a menos de $5 \mathrm{~g} / \mathrm{ave} / \mathrm{dia}$. Assim, esta quantidade reduzida do suplemento sendo retirado das dietas parece não ter efeito sobre o controle do consumo voluntário. Outros autores encontraram resultados semelhantes ao deste trabalho sobre consumo de 42 a 49 dias (Sayadi et al, 2005; Maiorka et al., 2002; Oba et al., 2012) e de 36 a 42 dias (Moravej et al., 2012a,b). Em contraste, Khajali et al. (2006) obtiveram maior consumo de ração com a retirada do premix de 42 a 56 dias. Entretanto, os autores não informaram quais fatores influenciaram no aumento do consumo, instigando apenas que o resultado não é consenso entre pesquisadores.

$O$ GPD dos frangos da dieta controle foi maior do que das aves 
submetidas às demais dietas. Como as vitaminas e os micro-minerais são essenciais no metabolismo celular (Boleli et al., 2002), atuando como cofatores em diversas reações aumentando a eficiência das vias de síntese do animal (Moravej et al., 2012a,b), entende-se que estes elementos necessitam estar presentes para o sucesso das reações metabólicas, as quais são fundamentais para a manutenção do GPD.

Nas criações industriais aumentar o GPD é um dos principais objetivos, pois reduz a idade de abate fazendo com que o retorno financeiro seja antecipado. Contudo, especula-se que as reservas de vitaminas e minerais no organismo, juntamente com a quantidade destes nutrientes presentes no milho e farelo de soja não sejam suficientes para fazer com que as aves tenham o mesmo rendimento em GPD. Semelhante a este resultado, Ferket e Qureshi (1992), Deyhim e Teeter (1993) e Patel et al. (1997) encontraram menor GPD em aves alimentadas sem a adição de premix na fase de terminação em relação as que receberam. Maiorka et al. (2002) encontrou menor peso de fígado (local de reserva de minerais e vitaminas) em frangos submetidos a restrição de premix de 42 a 49 dias, o que leva a crer que provavelmente as reservas hepáticas também não sejam suficientes para fazer com que as aves possam exprimir todo seu potencial genético para GPD. Esta analogia é feita em decorrência do resultado da análise de variância. Entretanto, a análise de regressão indica que o GPD piora com a antecipação da idade de retirada do premix. Alguns trabalhos demonstram que a retirada do premix na fase de terminação não afeta o GPD, como no estudo de Maiorka et al. (2002) que não encontraram diferenças com a retirada do premix aos 42, 45 e 49 dias, contudo estes autores indicaram uma tendência a uma melhora crescente no ganho daquelas aves que receberam ração com premix em relação as que não receberam. Khajali et al. (2006) de 42 a 56 dias e Oba et al. (2012) de 42 a 49 dias também não encontraram diferença no GPD, embora neste último a diferença real entre o grupo que recebeu suplemento e o que não recebeu tenha ficado em $33 \mathrm{~g} / \mathrm{ave}$ durante o período avaliado (42 a 49 dias).

$\mathrm{Na} \mathrm{CA}$ todas as idades de retirada do premix das dietas diferiram entre si, resultado corroborado pela análise de regressão que indicou um alto coeficiente de determinação $(r 2=0,965)$. Assim, a medida que avança a idade de retirada do premix piora a CA das aves. A CA é um fator determinado pela relação entre CR e GPD. Como não ocorreu diferença no consumo, o fator indicado como responsável pelo resultado é o GPD, já descrito anteriormente. Assim, fica implícito que com a retirada do suplemento mineral e vitamínico na fase de terminação, as aves convertem pior e tem menor eficiência na transformação do alimento em carne. A deficiência do suplemento provavelmente tem reflexo sobre 0 metabolismo do animal, o qual tem suas reações retardadas pela falta dos catalizadores, refletindo em uma menor velocidade de ganho de peso. Deyhim et al. (1995) enfoca que a suplementação de vitaminas e minerais pode aumentar a absorção de nutrientes e a utilização de alimentos.

Apesar de haver um número expressivo de trabalhos na literatura sobre o tema, os resultados da retirada do premix na fase de terminaçao são conflitantes. Enquanto Deyhim e Teeter (1993) Maiorka et al. (2002) Ebrahimnezhad et al. (2011) indicam que a retirada do suplemento aumenta a CA, enquanto que Skinner, et al. (1992), Cristmans et al. (1995), Khajali et al. (2006), Moravej et al. (2012a,b) reportam que a retirada não tem efeito sobre a $C A$. No entender de Skinner et al. (1992) o desempenho não é negativamente afetado, enquanto que para Shahrasb e 
Gerami (2011), Rahman, et al (2012) o desempenho é reduzido em aves alimentadas com dietas em que apenas o premix vitamínico foi retirado. Shahrasb et al $(2012 a, b)$ indicam que é possivel retirar os suplementos das dietas na fase de terminação sem que o desempenho seja afetado. Uma situação muito discutida e que implica na retirada do premix na ração de terminação é o possível comprometimento da qualidade da carne, porém os trabalhos de Moravej et al. (2012b) e Oba et al (2012) indicam que a qualidade nao é afetada pela retirada do suplemento na fase final.

Apesar do premix neste estudo reponder por apenas 1,3\% do custo total da ração, tanto a analise de variância quanto a análise de regressão do IBE indicam que quanto antes se retirar 0 premix da ração de terminação, pior é o IBE. Skinner, et al. (1992) e Oba et al. (2012) indicaram o contrário, que a retirada do premix é uma ferramenta para reduzir os custos de produção. Entretanto, o resultado deste trabalho indica que o custo na fase final tende a piorar com retirada antecipada do suplemento. Desta forma, torna-se inviável economicamente utilizar a estratégia da retirada do suplemento mineral e vitamínico da ração de terminação, e quanto mais dias se antecipa a retirada, mais elevado será o custo.

\section{CONCLUSÃO}

A retirada do suplemento mineral e vitamínico da ração de terminação de frangos de cortes reduz o desempenho zootécnico sem alterar o consumo alimentar, entretanto aumenta o custo de produção na fase de terminação.

\section{REFERÊNCIAS}

ARIKI, J.; SILVA, R.D.M. Retirada do suplemento e aditivos da alimentação final e seus efeitos no desempenho de frangos de corte. Anais do V Congresso Brasileiro de Avicultura, Fortaleza - Ce, 1977.

BOLELI, I.C.; MAIORKA, A.; MACARI, M. Estrutura funcional do trato digestório. In: MACARI, M.; FURLAN, R. L.;GONZALES, E. Fisiologia aviária aplicada a frangos de corte. 2. ed. Jaboticabal: FUNEP / UNESP, 2002. $375 p$

CHRISTMAS, R. B.; HARMS, R. H.; SLOAN, D. R. The absence of vitamins and trace minerals and broiler performance. Journal Applied Poultry Research, Champaigh, v. 4, n. 4, p. 407410, 1995.

\section{COBB. Manual de manejo de frangos Cobb 500: guia de manejo.} Suplemento: Desempenho e Nutriçao para frangos de corte. Cobb-Vantress Brasil, 2012. 14p.

DEYHIM, F.; TEETER, R. G. Dietary vitamin and/ or trace mineral premix effects on performance, humoral mediated immunity, and carcass composition of broilers during thermoneutral and high ambient temperature distress. Journal Applied Poultry Research. 2:347-355, 1993.

DEYHIM, F., STOEKER, B.S.; ADELEYE, B.G.; et al. The effect of the heat distress environment vitamin and trace mineral supplementation on performance, blood constituent, and tissue mineral concentration in broiler chicken. Nutrition Research, 15:521529. 1995.

EBRAHIMNEZHAD, Y.; HAJIHOSSEINI, R.; NAZERADL, K. et al. The Effects of Removal Mineral Premix at Different Periods of Rearing on Performance, Carcass Yield and Tibia Bone of Broiler Chicks. Pakistan Journal of Nutrition 10 (10): 930-934, 2011.

FERKET, P. R.; M. A. QURESHI. Performance and immunity of heatstressed broilers fed vitamin and 
eletrolyte supplemented drinking water. Poultry Science. 71:88-97. 1992.

GUIDONI, A.L.; GODOI, C.R. de M.; BELLAVER, C. Uso do índice nutricional bio-econômico como medida do desempenho nutricional. In: REUNIÃO ANUAL DA SOCIEDADE BRASILEIRA DE ZOOTECNIA, 31., 1994, Maringá, PR. Anais... Maringá: SBZ, 1994. p.32.

KHAJALI, F.; ASADI, K. E.; ZAMANI, M. Effect of vitamin and trace mineral withdrawal from finisher diets on growth performance and immunocompetence of broiler chickens. British Poultry Science, London, v. 47, n. 2, p. 159-162, 2006.

LEESON, S. Vitamin requirements: is there basis for reevaluating dietary specifications? World's Poultry Science Journal, v.63, n.2, p.255-266, 2007

MAIORKA, A.; LAURENTIZ, A.C.; SANTIN, E.; et al. Dietary vitamin or mineral mix removal during the finisher period on broiler chicken performance. Journal Applied Poultry Research ,11:121. 2002.

MORAVEJ, H.; ALAHYARI, S.M.; SHIVAZAD, M. Effects of the reduction or withdrawal of the vitamin premix from the diet on chicken performance and meat quality. Revista Brasileira de Ciência Avícola. v.14 n.4 Campinas. 2012a.

MORAVEJ, H.; ALAHYARI-SHAHRASB, M.; BAGHANI, M.R. et al. Withdrawal or reduction of the dietary vitamin premix on bone parameters of broiler chickens in two rearing systems. South African Journal of Animal Science, 42: 169$177,2012 b$

OBA, A; PINHEIRO, J. W; SILVA, C. A.; et al. Características produtivas e de qualidade de carne de frangos de corte submetidos a dietas de terminação sem suplemento vitamínico mineral ou calcário e fosfato bicálcico e submetidos a estresse térmico pré-abate. Semina:
Ciências Agrárias, Londrina, v. 33, suplemento 2, p. 3371-3378, 2012.

PATEL, K.P., EDWARDS III, M.H.; BAKER, D.H. Removal of vitamin and trace mineral supplements from broiler finisher diets. Journal Applied Poultry Research, 6: 191-198, 1997.

RAHMAN, M. A.; PARVIN, M.S.; SARKER, R.R. et al. Effects of growth promoter and multivitamin-mineral premix supplementation on body weight gain in broiler chickens. Journal of the Bangladesh Agricultural University, 10(2): 245-248, 2012.

ROSTAGNO, H.S.; ALBINO, L.F.T.; DONZELE, J.L. et al. Tabelas brasileiras para aves e suínos: composição de alimentos e exigências nutricionais. 3.ed. Viçosa, MG: UFV, Departamento de Zootecnia, 252p, 2011.

RUTZ F.; BERMUDEZ V. L.; PAN E. A. et al. Impacto da nutrição vitamínica sobre a resposta imunológica das aves. Anais III Simpósio Brasil Sul de avicultura 2002.

SAYADI, A. J.; NAVIDSHAD, B.; ABOLGHASEMI, A. et al. Effects of dietary mineral premix reduction or withdrawal on broilers performance. International Journal of Poultry Science, Pakistan, v. 4, n. 11, p. 896899, 2005.

SHAHRASB, M.A.; MORAVEJ, H.; SHIVAZAD, M. et al. Study of possible reduction or withdrawal of vitamin premix during finisher period in floor and battery cage broiler raising systems. African Journal of Biotechnology, 10: 63376341, 2011.

SHAHRASB, M. A.; MORAVEJ, H.; SHIVAZAD, $M$. Decreasing vitamin premix on chicken carcass composition and blood chemistry in floor and battery cage systems. Italian Journal of Animal Science, 11:14, 2012. 
SKINNER, J.T.; WALDROUP, A.L; WALDROUP, P.W. Effect of removal of vitamin and trace mineral supplements from grower and finisher diets on live performance and carcass composition of broilers. Journal Applied Poultry Research, 1: 280-286, 1992. 